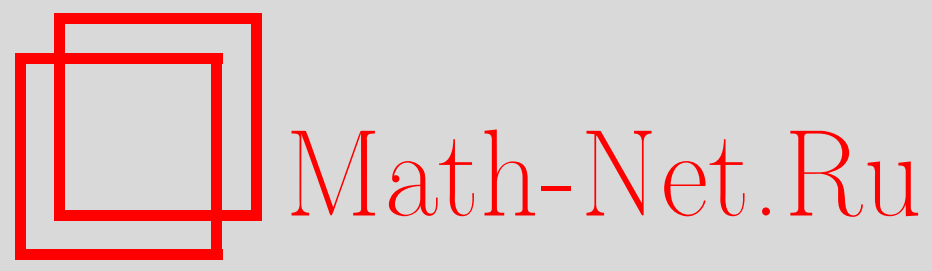

Т. Д. Блайвас, Функция Шеннона сложности интервального поиска на булевом кубе в классе деревьев, Дискрет. матем., 2006, том 18, выпуск 2, 111-122

DOI: https://doi.org/10.4213/dm50

Использование Общероссийского математического портала Math-Net.Ru подразумевает, что вы прочитали и согласны с пользовательским соглашением http://www . mathnet.ru/rus/agreement

Параметры загрузки:

IP : 54.89 .56 .158

26 апреля 2023 г., 15:28:13

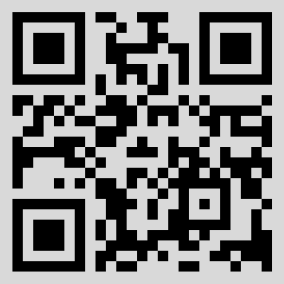




\title{
Функция Шеннона сложности интервального поиска на булевом кубе в классе деревьев
}

\author{
() 2006 г. Т. Д. Блайвас
}

\begin{abstract}
Для задачи интервального поиска на булевом кубе исследуется поведение функции Шеннона сложности в классе древовидных схем, называемых информационными деревьями. Показано, что для баз данных, чья мощность совпадает по порядку с мощностью булева куба, функция Шеннона по порядку равна оптимальной сложности в классе сбалансированных древовидных схем. Для баз данных с мощностью, меньшей по порядку, чем мощность булева куба, найдена асимптотика логарифма функции Шеннона.
\end{abstract}

\section{1. Введение}

В работе исследуется следующая задача информационного поиска, называемая задачей интервального поиска на булевом кубе. Имеется некоторое подмножество $V n$-мерного булева куба $B_{2}^{n}$, называемое библиотекой. На булевом кубе берется произвольный интервал $(u, w)$, где $u=\left(u_{1}, \ldots, u_{n}\right), w=\left(w_{1}, \ldots, w_{n}\right)$ и $u \preccurlyeq w$, то есть $u_{i} \leqslant w_{i} i=1, \ldots, n$. Требуется определить все элементы $y \in V, y=\left(y_{1}, \ldots, y_{n}\right)$, называемые записями, такие, что $u \preccurlyeq y \leqslant w$.

Задача интервального поиска на булевом кубе соответствует поиску по маске. Те компоненты с номером $i$, для которых $u_{i}=w_{i}$, задают маску, и в этих компонентах значения у искомых объектов должны совпадать с маской, то есть $y_{i}=u_{i}=w_{i}$. А для тех $i$, для которых $0=u_{i} \neq w_{i}=1$, компонента $y_{i}$ может принимать произвольное значение из $\{0,1\}$.

Задачу можно решить, если на каждом шаге алгоритма проверять условие $u_{j} \leqslant y_{j} \leqslant w_{j}$ для некоторого фиксированного индекса $j \in\{1, \ldots, n\}$. Произвольный алгоритм поиска такого рода можно представить в виде так называемого информационного дерева. Сложность информационного дерева вводится так же, как в [1], и соответствует среднему времени работы соответствующих алгоритмов.

В классе всех деревьев исследовалось поведение функции Шеннона сложности для задачи интервального поиска на булевом кубе. Верхние оценки достигаются в классе сбалансированных деревьев. При $k \geqslant 2^{n-1}$ получено точное значение функции Шеннона. При $k \asymp 2^{n}$ получены оценки, которые задают порядок функции Шеннона и показывают, что при данных ограничениях на размер библиотеки значение функщии Шеннона по порядку совпадает со сложностью сбалансированных деревьев $k^{\log _{2} 4 / 3}$ (см. [1]). При $\log _{2} \log _{2} n=\bar{o}\left(\log _{2} k\right)$ найдена асимптотика логарифма функции Шеннона, равная $\log _{2}(4 / 3) \log _{2} k$. 


\section{2. Основные понятия и формулировка результатов}

Мы будем использовать терминологию и обозначения из работы [2], но поскольку в данной работе рассматриваются только древовидные схемы, здесь будет приведена несколько упрощенная версия понятия информационного графа.

Если $X$ - множество символов запросов с заданным на нем вероятностным пространством $\langle X, \sigma, \mathbf{P}\rangle$, где $\sigma$ - алгебра подмножеств множества $X, \mathbf{P}$ - вероятностная мера на $\sigma, Y$ - множество символов данных (записей), $\rho$ - бинарное отношение на $X \times Y$, называемое отношением поиска, то пятерка $S=\langle X, Y, \rho, \sigma, \mathbf{P}\rangle$ называется типом. Тройка $I=\langle X, V, \rho\rangle$, где $V$ - некоторое конечное подмножество множества $Y$, называемое библиотекой, называется задачей информационного поиска (ЗИП) типа $S$. Содержательно ЗИП $I=\langle X, V, \rho\rangle$ состоит в перечислении для произвольно взятого запроса $x \in X$ всех тех и только тех записей $y \in V$, для которых $x \rho y$. Если F्F - множество символов одноместных предикатов, определенных на $X$, то $\mathscr{F}$ называется базовым множеством и описывает множество элементарных операций, используемых при решении задачи информащионного поиска.

Над базовым множеством F्F определим понятие информационного графа (ИГ). В конечной многополюсной ориентированной сети выбирается вершина (полюс), называемая корнем. Остальные полюсы называются листьями и им приписываются записи из $Y$. Ребрам ИГ приписываются предикаты из множества $\mathscr{F}$. Таким образом нагруженную многополюсную ориентированную сеть называют информационным графом над базовым множеством F्F. Затем определяется функционирование ИГ. Предикатное ребро проводит запрос $x \in X$, если предикат ребра истинен на $x$; ориентированная цепочка ребер проводит $x$, если каждое ребро цепочки проводит $x$; запрос $x$ проходит в вершину $\beta$ ИГ, если существует ориентированная цепь, ведущая из корня в вершину $\beta$, которая проводит $x$; запись $y$, приписанная листу $\alpha$, попадает в ответ ИГ на $x$, если $x$ проходит в лист $\alpha$. Ответом $И Г U$ на запрос $x$ называют множество записей, попавших в ответ $U$ на $x$, и обозначают его $\mathscr{F}_{U}(x)$. Эту функщию $\mathscr{I}_{U}(x)$ считают результатом функционирования ИГ $U$.

ИГ $U$ решает ЗИП $I=\langle X, V, \rho\rangle$, если $\mathscr{I}_{U}(x)=\{y \in V: x \rho y\}$.

Вводится сложность ИГ. Предикат $\varphi_{\beta}(x)$, истинный на $x$, если $x$ проходит в вершину $\beta$, и ложный в противном случае, называется функцией фильтра вершины $\beta$. Сложностью ИГ $U$ на запросе $x \in X$ называется число

$$
T(U, x)=\sum_{\beta \in \mathscr{R}} \psi_{\beta} \varphi_{\beta}(x),
$$

где $\mathscr{R}$ - множество вершин ИГ $U, \psi_{\beta}$ - число ребер, исходящих из вершины $\beta$. Эта величина равна числу функщий, вычисленных алгоритмом поиска, определяемым ИГ $U$, на запросе $x$.

Если каждая функщия из $\mathscr{F}$ измерима (относительно алгебры $\sigma$ ), то для любого ИГ $U$ над $\mathscr{F}$ функция $T(U, x)$ измерима.

Сложностью ИГ $U$ называется математическое ожидание величины $T(U, x)$, равное $T(U)=M_{x} T(U, x)$. Она характеризует среднее время поиска. Если $f$ - предикат на множестве $X$, то $N_{f}(x)=\{x \in X: f(x)=1\}$.

Легко показать, что

$$
T(U)=\sum_{\beta \in U} \psi_{\beta} \mathbf{P}\left(N_{\varphi_{B}}(x)\right)
$$


Число $\mathbf{P}\left(N_{\varphi_{\beta}}(x)\right)$ назовем сложностью ребра, исходящего из вершины $\beta$. Согласно (1), сложность ИГ равна сумме сложностей ребер.

Рассмотрим следующую ЗИП. Имеется некоторое $k$-элементное подмножество $n$-мерного булева куба $V \in B_{2}^{n}$ (библиотека). На булевом кубе задан некоторый интервал $(u, w)$, где $u=\left(u_{1}, \ldots, u_{n}\right), w=\left(w_{1}, \ldots, w_{n}\right)$, и $u \leqslant w$, то есть $u_{i} \leqslant w_{i}, i=1, \ldots, n$. Требуется определить все элементы $y \in V$, удовлетворяющие условию $u \preccurlyeq y \preccurlyeq w$.

Очевидно, что если $u_{i}=1$ для некоторого $i$, то и $w_{i}=1$, а следовательно, и $y_{i}=1$. Аналогично, если $w_{i}=0$, то и $y_{i}=0$. Таким образом, вышеописанная ЗИП сводится к следующей: есть библиотека $V \in B_{2}^{n},|V|=k$, берем запрос $x=\left(x_{1}, \ldots, x_{n}\right)-$ трехзначный вектор, компоненты которого могут быть равны либо 1 , либо 0 , либо 2: если $u_{i}=1$, то $x_{i}=1$, если $w_{i}=0$, то $x_{i}=0$, в противном случае $x_{i}=2$. Для данного запроса $x=\left(x_{1}, \ldots, x_{n}\right)$ нужно найти все $y=\left(y_{1}, \ldots, y_{n}\right) \in V$, для которых $y_{i}=x_{i}$, если $x_{i}=1$ или $x_{i}=0$, и $y_{i}$-любое из $\{0,1\}$, если $x_{i}=2$.

Получаем тип задач $S_{n}=\left\langle B_{3}^{n}, B_{2}^{n}, \rho, \sigma, P\right\rangle$, где $B_{3}^{n}=\{0,1,2\}^{n}$ и $B_{2}^{n}=\{0,1\}^{n}-$ трехзначный и двузначный (булев) кубы, соответственно, $\sigma$ - множество подмножеств $B_{3}^{n}, \rho: x \rho y \Longleftrightarrow\left(x_{i}=y_{i}\right) \vee\left(x_{i}=2\right)$, и $\mathbf{P}$ - равномерная вероятностная мера на $B_{3}^{n}$, то есть $\mathbf{P}(x)=3^{-n}$ для любого $x \in B_{3}^{n}$.

Множество задач $I=\left\langle B_{3}^{n}, V, \rho\right\rangle$ типа $S_{n}$, где $|V|=k$, обозначим через $\mathscr{I}(n, k)$.

Пусть $x \in\{0,1,2\}$ и $y \in\{0,1\}$. Определим функщию $x^{y}$, полагая

$$
x^{y}= \begin{cases}x, & \text { если }(y=1) \&(x \neq 2), \\ \bar{x}, & \text { если }(y=0) \&(x \neq 2), \\ 1, & \text { если } x=2,\end{cases}
$$

причем $\bar{x}$ понимается здесь как булево отрицание.

Вершину со степенью полуисхода, равной нулю, назовем висячей вершиной. Информационным деревом (ИД) назовем ИГ без циклов, множество листьев которого совпадает с множеством висячих вершин и все ребра которого ориентированы от корня к листьям. Высотой ИД назовем длину максимального пути из корня в лист.

Будем рассматривать базис переменных $\mathscr{F}=\left\{x_{i}^{s_{i}} \mid s_{i} \in\{0,1\}, i=1, \ldots, n\right\}$. Через $\mathscr{D}_{I}$ обозначим всевозможные информационные деревья над базисом $\mathscr{F}$, решающие ЗИП $I \in \mathscr{I}(n, k)$. Введем обозначение $T(I)=\min _{D \in \mathscr{D}} T(D)$. Функцией Шеннона сложности в классе древовидных схем над базисом переменных назовем число

$$
L(n, k)=\max _{I \in \Phi(n, k)} T(I) .
$$

Теорема 1. Пусть $n \rightarrow \infty, k \rightarrow \infty$, тогда

(1) если $k \geqslant 2^{n-1}$, то $L(n, k)=6\left(\frac{4}{3}\right)^{n-1}+k\left(\frac{2}{3}\right)^{n-1}-6$,

(2) если $k \asymp 2^{n}$, то $L(n, k) \asymp k^{\log _{2}(4 / 3)}$,

(3) если $\log _{2} \log _{2} n=\bar{o}\left(\log _{2} k\right)$, то $\log _{2} L(n, k) \sim \log _{2}(4 / 3) \log _{2} k$.

\section{3. Верхняя оценка}

Скажем, что вершина дерева находится на ярусе с номером $i$, если длина пути от корня до этой вершины равна $i$. Будем говорить, что ребро находится на ярусе с номером $i$, если оно исходит из вершины яруса с номером $i-1$. 
Пусть $\sigma=\left(i_{1}, \ldots, i_{n}\right) \in S_{n}-$ некоторая перестановка, действующая на множестве, состоящем из первых $n$ натуральных чисел. Сбалансированным деревом с характеристикой $m$, соответствующим перестановке $\sigma$, будем называть такое дерево, что из каждой вершины яруса с номером $p \leqslant m$ выходит точно два ребра. Для каждой вершины яруса с номером $p$, меньшим $m$, ребрам, исходящим из этой вершины, приписаны переменные $x_{i_{p}}^{0}$ и $x_{i_{p}}^{1}$. Из некоторых вершин яруса с номером $m+1$ исходит не более $2^{n-m}$ цепочек ребер, причем функции проводимости этих цепочек различны для каждой пары цепочек ребер, исходящих из одной вершины, и имеют вид $x_{i_{m+1}}^{\sigma_{1}} \& \ldots \& x_{i_{n}}^{\sigma_{n-m}}$, где $\sigma_{j} \in\{0,1\}$, $j=1, \ldots, n-m$.

Замечание 1. Очевидно, что для любой ЗИП $I \in \mathscr{I}(n, k)$ существуют сбалансированные деревья, решающие ЗИП $I$.

Лемма 1. Пусть $D$ - сбалансированное дерево высоты $n$ с $k$ концевыми цепочками и характеристикой $\left[\log _{2} k\right]$. Тогда

$$
T(D)=6\left(\frac{4}{3}\right)^{\left[\log _{2} k\right]}+3 k\left(\frac{2}{3}\right)^{\left[\log _{2} k\right]}-6-3 k\left(\frac{2}{3}\right)^{n} \text {. }
$$

Доказательство. Для каждого номера яруса $i \in\left\{1, \ldots,\left[\log _{2} k\right]\right\}$ дерево $D$ имеет $2^{i}$ ребер, а для каждого $i \in\left\{\left[\log _{2} k\right]+1, \ldots, n\right\}-k$ ребер. Вероятность попасть в концевую вершину ребра яруса с номером $i \in\{1, \ldots, n\}$ равна $(2 / 3)^{i}$. Следовательно,

$$
\begin{aligned}
T(D) & =\sum_{i=1}^{\left[\log _{2} k\right]}\left(\frac{2}{3}\right)^{i-1} 2^{i}+\sum_{i=\left[\log _{2} k\right]+1}^{n}\left(\frac{2}{3}\right)^{i-1} k \\
& =2 \sum_{i=1}^{\left[\log _{2} k\right]}\left(\frac{4}{3}\right)^{i-1}+k\left(\frac{2}{3}\right)^{\left[\log _{2} k\right]} \sum_{i=1}^{n-\left[\log _{2} k\right]}\left(\frac{2}{3}\right)^{i-1} \\
& =6\left(\left(\frac{4}{3}\right)^{\left[\log _{2} k\right]}-1\right)+3 k\left(\left(\frac{2}{3}\right)^{\left[\log _{2} k\right]}-\left(\frac{2}{3}\right)^{n}\right) \\
& =6\left(\frac{4}{3}\right)^{\left[\log _{2} k\right]}+3 k\left(\frac{2}{3}\right)^{\left[\log _{2} k\right]}-6-3 k\left(\frac{2}{3}\right)^{n} .
\end{aligned}
$$

Лемма 1 доказана.

Рассмотрим функцию

$$
A(a)=2 a^{\log _{2}(4 / 3)}+a^{\log _{2}(2 / 3)} .
$$

Лемма 2. Справедливы равенства

$$
\max _{a \in[1 / 2 ; 1]} A(a)=A(1 / 2)=A(1)=3 .
$$

Доказательство. Исследуем функщию

$$
A(a)=2 a^{\log _{2}(4 / 3)}+a^{\log _{2}(2 / 3)} .
$$

Ясно, что

$$
A\left(\frac{1}{2}\right)=2 \cdot \frac{3}{4}+\frac{3}{2}=3, \quad A(1)=2+1=3
$$


Нетрудно видеть, что

$$
\begin{aligned}
& A^{\prime}(a)=2 \log _{2}(4 / 3) a^{\log _{2}(2 / 3)}+\log _{2}(2 / 3) a^{\log _{2}(1 / 3)}, \\
& A^{\prime}(\hat{a})=0 \Longleftrightarrow a^{\log _{2}(1 / 3)}\left(2 \log _{2}(4 / 3) \hat{a}+\log _{2}(2 / 3) \Longleftrightarrow \hat{a}=\frac{1}{2} \log _{4 / 3}(3 / 2),\right. \\
& A(\hat{a})=(\hat{a})^{\log _{2}(2 / 3)}(2 \hat{a}+1)=(3 / 2)\left(\log _{4 / 3} \frac{3}{2}\right)^{\log _{2}(3 / 2)} \log _{4 / 3} 2 \approx 2,956 .
\end{aligned}
$$

Таким образом, функция $A(a)$ принимает максимальное значение, равное 3 , на границах отрезка $[1 / 2,1]$.

Лемма 3. Пусть $I \in \mathscr{I}(n, k), D-$ сбалансированное дерево высоты $n c k$ конщевыми вериинами и характеристикой $\left[\log _{2} k\right]$. Тогда

$$
T(D) \leqslant 9 k^{\log _{2}(4 / 3)}-3 k\left(\frac{2}{3}\right)^{n}-6 .
$$

Доказательство. Пусть сбалансированное дерево $D$ имеет характеристику $\left[\log _{2} k\right]$. В силу леммы 1

$$
T(D)=6\left(\frac{4}{3}\right)^{\left[\log _{2} k\right]}+3 k\left(\frac{2}{3}\right)^{\left[\log _{2} k\right]}-6-3 k\left(\frac{2}{3}\right)^{n}
$$

Пусть $\left[\log _{2} k\right]=\log _{2}(a k), a \in[1 / 2,1]$. Тогда

$$
\begin{aligned}
T(D) & =6\left(\frac{4}{3}\right)^{h}+3 k\left(\frac{2}{3}\right)^{h}-6-3 k\left(\frac{2}{3}\right)^{n} \\
& =6(a k)^{\log _{2}(4 / 3)}+3 k(a k)^{\log _{2}(2 / 3)}-6-3 k\left(\frac{2}{3}\right)^{n} \\
& =3 k^{\log _{2}(4 / 3)}\left(2 a^{\log _{2}(4 / 3)}+a^{\log _{2}(2 / 3)}\right)-6-3 k\left(\frac{2}{3}\right)^{n}
\end{aligned}
$$

то есть

$$
T(D)=3 A(a) k^{2-\log _{2} 3}-6-3 k\left(\frac{2}{3}\right)^{n} .
$$

Из леммы 2 следует, что

$$
T(D) \leqslant 9 k^{\log _{2}(4 / 3)}-3 k\left(\frac{2}{3}\right)^{n}-6
$$

Следствие 1. Справедливы оченки

$$
\begin{aligned}
& L(n, k) \leqslant 6(4 / 3)^{\left[\log _{2} k\right]}+3 k(2 / 3)^{\left[\log _{2} k\right]}-6-3 k(2 / 3)^{n}, \\
& L(n, k)<9 k^{\log _{2}(3 / 4)} .
\end{aligned}
$$




\section{4. Нижняя оценка}

Лемма 4. При $k \geqslant 2^{n-1}$ существует такая библиотека $V_{n, k} \subseteq B_{2}^{n},\left|V_{n, k}\right|=k$, что в любом подкубе размерности $i \geqslant 1$, находится не менее $2^{i-1}$ и не более $2^{i}$ записей из библиотеки.

Доказательство. Библиотеку $V_{n, 2^{n-1}}$ строим следующим образом. $B_{2}^{n}$ содержит в точности $2^{n-1}$ записей с четным числом единиц. Пусть библиотека $V_{n, 2^{n-1}}$ состоит из всех таких записей. В каждом подкубе размерности 1 находится точно один элемент с четным числом единиц. Следовательно, так как каждый подкуб $B_{i}$ размерности $i$ содержит $2^{i-1}$ подкубов размерности 1 , в подкубе $B_{i}$ находится точно $2^{i-1}$ записей из библиотеки. Библиотеку $V_{n, k}, k>2^{n-1}$, получим, если добавим к записям библиотеки $V_{n, 2^{n-1}}$ произвольные $k-2^{n-1}$ различных записей, не содержащихся в $V_{n, 2^{n-1}}$. Очевидно, что в каждом подкубе размерности $i \geqslant 1$, содержатся записи из $V_{n, k}$, причем их не менее $2^{i-1}$ и не более $2^{i}$.

Лемма 5. $\Pi p u k \geqslant 2^{n-1}$

$$
L(n, k) \geqslant 6\left(\frac{4}{3}\right)^{n-1}+k\left(\frac{2}{3}\right)^{n-1}-6 .
$$

Доказательство. Возьмем библиотеку $V_{n, k}$, построенную, как в лемме 4. Пусть дерево $D$ решает задачу $I=\left\langle B_{3}^{n}, V_{n, k}, \rho\right\rangle$ над базисом переменных. В дереве $D$ каждая цепочка длины $s \leqslant n-1$, выходящая из корня, определяет в кубе $B_{2}^{n}$ некоторый подкуб размерности $n-s$. А именно, если цепочке $c$ приписана суммарная конъюнкция $x_{i_{1}}^{\sigma_{i_{1}}} \& \ldots \& x_{i_{s}}^{\sigma_{i_{s}}}$, то к конечной вершине цепочки из корня проходит каждый запрос $x=\left(x_{1}, \ldots, x_{n}\right)$, для которого $x_{i},=\sigma_{i j}, j=1, \ldots, s$; напротив, если для запроса $x=\left(x_{1}, \ldots, x_{n}\right)$ существует $j \in\{1, \ldots, s\}$, для которого $x_{i j} \neq 2$ и $x_{i j} \neq \sigma_{i j}$, то запрос $x$ не проходит в концевую вершину цепочки $c$.

Таким образом, каждая концевая вершина яруса с номером $i$ проводит запрос к записям из подкуба, размерность которого $n-i$. Если к ярусу вершин с номером $i \leqslant n-1$ ведут менее чем $2^{i}$ цепочек, то число всех записей из $V_{n, 2^{n-1}} \subseteq V_{n, k}$, к которым потенциально может пройти запрос, менее чем $2^{i} \cdot 2^{n-i-1}=2^{n-1}$, что противоречит допустимости ИД $D$. Следовательно, на всех ярусах с номерами $i=1, \ldots, n-1$ в решающем дереве $D$ находится не менее $2^{i}$ ребер. На последнем ярусе в решающем дереве не могут находиться менее $k$ ребер. Обозначим через $E_{i}$ число ребер на ярусе с номером $i$. Тогда

$$
\begin{aligned}
T(D) & =\sum_{i=1}^{n} E_{i}\left(\frac{2}{3}\right)^{i-1} \geqslant 2+\frac{2}{3} 2^{2}+\ldots+\left(\frac{2}{3}\right)^{n-2} 2^{n-1}+\left(\frac{2}{3}\right)^{n-1} k \\
& =2\left(1+\frac{4}{3}+\ldots+\left(\frac{4}{3}\right)^{n-2}\right)+\left(\frac{2}{3}\right)^{n-1} k=6\left(\left(\frac{4}{3}\right)^{n-1}-1\right)+\left(\frac{2}{3}\right)^{n-1} k
\end{aligned}
$$

Следовательно,

$$
L(n, k) \geqslant 6(4 / 3)^{n-1}+k(2 / 3)^{n-1}-6
$$

Лемма 6. Для любых натуральных $n, k, 3 И П ~ I \in \Phi(n, k)$ справедливо неравенство

$$
T(I) \geqslant 6 \frac{k}{2^{n}}\left(\left(\frac{4}{3}\right)^{n}-1\right) .
$$


Доказательство. Пусть $D$ - произвольное дерево, решающее ЗИП $I$. Обозначим через $E_{i}$ число ребер на ярусе с номером $i$ в дереве $D$. По определению сложности

$$
T(D)=\sum_{i=1}^{n} E_{i}(2 / 3)^{i-1}
$$

Оценим снизу числа $E_{i}, i=1, \ldots, n$. Заметим, что если вершина $\beta$ является концом некоторой цепочки ребер $c$ длины $i$, выходящей из корня, то число записей, к которым могут пройти запросы через вершину $\beta$, может быть не более чем $2^{n-i}$. Точнее, если цепь $c$ имеет суммарную нагрузку $x_{m_{1}}^{s_{m_{1}}} \& \ldots \& x_{m_{i}}^{s_{m_{i}}}$, то запросы через вершину $\beta$ могут пройти только к записям, у которых компоненты с номерами $m_{1}, \ldots, m_{i}$ равны $s_{m_{1}}, \ldots, s_{m_{i}}$ соответственно, а остальные компоненты принимают значения из $\{0,1\}$. Предположим, что $E_{i}<k / 2^{n-i}$. Тогда число записей, достижимых из всех вершин яруса с номером $i$ (то есть достижимых из корня), не больше, чем

$$
E_{i} 2^{n-i}<\frac{k}{2^{n-i}} 2^{n-i}=k
$$

а это означает, что число достижимых из корня записей строго меньше числа $k$ элементов библиотеки в задаче $I$. Следовательно, дерево $D$ не решает ЗИП $I$, что противоречит выбору дерева $D$. Значит, $E_{i} \geqslant k / 2^{n-i}$. Тогда

$$
\begin{aligned}
T(D) & =\sum_{i=1}^{n} E_{i}\left(\frac{2}{3}\right)^{i-1} \geqslant \sum_{i=1}^{n} \frac{k}{2^{n}} 2^{i}\left(\frac{2}{3}\right)^{i-1} \\
& =2 \frac{k}{2^{n}} \sum_{i=1}^{n}\left(\frac{4}{3}\right)^{i-1}=6 \frac{k}{2^{n}}\left(\left(\frac{4}{3}\right)^{n}-1\right) .
\end{aligned}
$$

Пусть $V_{n}$ - последовательность библиотек, а $s_{n}$ - неубывающая последовательность натуральных чисел такая, что $\left|V_{n}\right| 2^{-s_{n}} \rightarrow \infty$ при $n \rightarrow \infty$. Будем говорить, что последовательность библиотек $V_{n}$ является равномерно распределенной порядка $s_{n}$, если в любом $\left(n-s_{n}\right)$-мерном подкубе булева куба $B_{2}^{n}$ число записей из $V_{n}$ асимптотически равно $\left|V_{n}\right| 2^{-s_{n}}$ при $n \rightarrow \infty$.

Лемма 7. Пусть $V_{n}$ - последовательность равномерно распределенных библиотек порядка $s_{n}, t_{n}-$ неубывающая последовательность такая, что $t_{n}<s_{n}$. Тогда $V_{n}$ является последовательностью равномерно распределенных библиотек порядка $t_{n}$.

Доказательство. Каждый подкуб размерности $n-t_{n}$ содержит $2^{n-t_{n}} / 2^{n-s_{n}}=2^{s_{n}-t_{n}}$ непересекающихся подкубов размерности $2^{n-s_{n}}$. В каждом из этих подкубов размерности $2^{n-s_{n}}$ содержится асимптотически $\left|V_{n}\right| 2^{-s_{n}}$ элементов библиотеки. Следовательно, в подкубе размерности $n-t_{n}$ содержится асимптотически $\left|V_{n}\right| 2^{-s_{n}} 2^{s_{n}-t_{n}}=\left|V_{n}\right| 2^{-t_{n}}$ элементов из библиотеки $V_{n}$.

Лемма 8. Пусть $V_{n}$ - последовательность равномерно распределенных библиотек порядка $s_{n}, s_{n} \rightarrow \infty, u I_{n}=\left\langle B_{3}^{n}, V_{n}, \rho\right\rangle$ - соответствующая последовательность ЗИП. Тогда при $n \rightarrow \infty$

$$
T\left(I_{n}\right) \gtrsim 6\left(\frac{4}{3}\right)^{s_{n}}
$$


Доказательство. Пусть дерево $D_{n}$ решает задачу $I_{n}$ над базисом переменных. В дереве $D_{n}$ каждая цепочка длины $s \leqslant s_{n}$, выходящая из корня, определяет в кубе $B_{2}^{n}$ некоторый подкуб размерности $n-s$. Именно, если цепочке $c$ приписана суммарная конъюнкция $x_{i_{1}}^{\sigma_{i_{1}}} \& \ldots \& x_{i_{s}}^{\sigma_{i_{s}}}$, то к конечной вершине цепочки из корня проходит каждый запрос $x=\left(x_{1}, \ldots, x_{n}\right)$, для которого $x_{i_{j}}=\sigma_{i_{j}}, j=1, \ldots, s$; напротив, если для запроса $x=\left(x_{1}, \ldots, x_{n}\right)$ существует $j \in\{1, \ldots, s\}$, для которого $x_{i j} \neq 2$ и $x_{i_{j}} \neq s_{i j}$, то запрос $x$ не проходит в концевую вершину цепочки $c$. Заметим, что в каждом подкубе размерности $n-s$ содержится не более чем $\left|V_{n}\right| 2^{-s}+\bar{o}\left(\left|V_{n}\right| 2^{-s}\right)$ элементов библиотеки $V_{n}$. Это значит, что из концевой вершины цепочки длины $s$ достижимы не более чем $\left|V_{n}\right| 2^{-s}+\bar{o}\left(\left|V_{n}\right| 2^{-s}\right)$ записей библиотеки. Если $c(s, n)$ - число цепочек длины $s$, начинающихся в корне дерева $D_{n}$, то число достижимых из корня записей не более, чем $c(s, n)\left(\left|V_{n}\right| 2^{-s}+\bar{o}\left(\left|V_{n}\right| 2^{-s}\right)\right)$. Если $c(s, n)=\bar{o}\left(2^{s}\right)$, то, очевидно, число достижимых из корня записей есть $\bar{o}\left(\left|V_{n}\right|\right)$, что противоречит допустимости ИД $D_{n}$. Следовательно, для любого $s \leqslant s_{n}$ число ребер на ярусе с номером $s$ асимптотически равно $2^{s}$.

Оценим сложность дерева $D_{n}$. Обозначим через $E_{i}$ число ребер на ярусе с номером i. Тогда

$$
\begin{aligned}
T\left(D_{n}\right) & =\sum_{i=1}^{n}\left(\frac{2}{3}\right)^{i-1} E_{i} \geqslant \sum_{i=1}^{s_{n}}\left(\frac{2}{3}\right)^{i-1} E_{i} \\
& =\frac{3}{2} \sum_{i=1}^{s_{n}}\left(\frac{2}{3}\right)^{i}\left(2^{i}+\bar{o}\left(2^{i}\right)\right)=\frac{3}{2} \sum_{i=1}^{s_{n}}\left(\left(\frac{4}{3}\right)^{i}+\bar{o}\left(\left(\frac{4}{3}\right)^{i}\right)\right) \\
& \sim \frac{3}{2} \sum_{i=1}^{s_{n}}\left(\frac{4}{3}\right)^{i}=6\left(\left(\frac{4}{3}\right)^{s_{n}}-1\right) \sim 6\left(\frac{4}{3}\right)^{s_{n}}
\end{aligned}
$$

при $n \rightarrow \infty$.

Обозначим через $\mathscr{V}(n, k, s, \alpha)$ множество библиотек мощности $k$ таких, что в любом подкубе размерности $n-s$ содержится больше, чем $\left[k 2^{-s}\right]-\alpha$, и меньше, чем $\left[k 2^{-s}\right]+\alpha$ записей.

В работах $[2,3]$ была сформулирована и доказана следующая теорема.

Лемма 9. Пусть $s(n) \rightarrow \infty, k(n) 2^{-s(n)} \rightarrow \infty, \alpha(n)=\bar{o}\left(k(n) 2^{-s(n)}\right), k(n)=\bar{o}\left(2^{n}\right)$. Тогдa

$$
\begin{aligned}
& |\mathcal{V}(n, k(n), s(n), \alpha(n))| \\
& \qquad\left(\begin{array}{c}
2^{n} \\
k
\end{array}\right)\left(1-\exp \left(2 s \ln n+\ln k-\frac{\alpha^{2} 2^{s}}{2 k}+\bar{o}\left(\frac{\alpha^{2} 2^{s}}{k}\right)+\underline{o}\left(\frac{k^{3}}{4^{n}}\right)\right)\right) .
\end{aligned}
$$

Лемма 10. Пусть при $n \rightarrow \infty, k(n) \rightarrow \infty, \log _{2} k(n) \lesssim c n, c<2 / 3$,

$$
\log _{2} \log _{2} n=\bar{o}\left(\log _{2} k(n)\right), \quad f(n, k)=\left(16 \log _{2} k \ln n\right)^{\delta}, \quad \delta>1 \text {. }
$$

Тогда существует $N$ такое, что для любого $n \geqslant N$ существует последовательность равномерно распределенных библиотек порядка

$$
s(n, k)=\left[\log _{2} k(n)-\log _{2} f(n, k)\right]
$$

и мочности $k(n)$. 
Доказательство. Отметим, что

$$
\log _{2} f(n, k)=\delta\left(\log _{2} \log _{2} k(n)+\log _{2} \ln n+4\right)=\bar{o}\left(\log _{2} k(n)\right) .
$$

Положим

$$
\alpha(n, k)=(f(n, k))^{\varepsilon},
$$

где $\varepsilon=(1 / 2)(1+1 / \delta)<1$. Тогда $\alpha(n, k)=\bar{o}\left(k(n) 2^{-s(n, k)}\right)$. Введем обозначения

$$
\begin{aligned}
& A=2 s(n, k) \ln n+\ln k(n)-\frac{\alpha^{2}(n, k) 2^{s(n, k)}}{2 k(n)}, \\
& B=\bar{o}\left(\frac{\alpha^{2}(n, k) 2^{s(n, k)}}{k(n)}\right)+\underline{O}\left(\frac{k^{3}(n)}{4^{n}}\right) .
\end{aligned}
$$

Оценим $A$ и $B$. Нетрудно видеть, что

$$
\begin{aligned}
A & \leqslant 2 \ln n\left(\log _{2} k(n)-\log _{2} f(n, k)\right)+\ln k-\frac{f(n, k)^{1+1 / \delta} k(n)}{2 k(n) f(n, k)} \\
& =2 \ln n \log _{2} k(1+\bar{o}(1))+\ln k-\frac{1}{4} f(n, k)^{1 / \delta} \\
& \leqslant 3 \ln n \log _{2} k(1+\bar{o}(1))-4 \ln n \log _{2} k=-\ln n \log _{2} k(1+\bar{o}(1))<0, \\
B & =\bar{o}\left(\frac{\alpha^{2}(n, k) 2^{s(n, k)}}{k(n)}\right)+\underline{O}\left(\frac{k^{3}(n)}{4^{n}}\right) \\
& \leqslant \bar{o}\left(\frac{f(n, k)^{1+\frac{1}{\delta} k(n)}}{2 k(n) f(n, k)}\right)+\underline{O}\left(\frac{2^{3 c n}}{4^{n}}\right)=\bar{o}\left(\log _{2} k \ln n\right)+\bar{o}(1) .
\end{aligned}
$$

Таким образом, при достаточно больших $n$ и $k(n) \lesssim c n, c<2 / 3$

$$
\begin{aligned}
& 2 s(n, k) \ln n+\ln k(n)-\frac{\alpha^{2}(n, k) 2^{s(n, k)}}{2 k(n)}+\bar{o}\left(\frac{\alpha^{2}(n, k) 2^{s(n, k)}}{2 k(n)}\right)+\underline{o}\left(\frac{k^{3}(n)}{4^{n}}\right) \\
&=A+B<-\log _{2} k \ln n(1+\bar{o}(1))+\bar{o}\left(\log _{2} k \ln n\right)+\bar{o}(1)<0,
\end{aligned}
$$

и при достаточно больших $n$ как следствие леммы 9 и неравенства 2 , получаем, что

$$
|\mathcal{V}(n, k(n), s(n, k), \alpha(n, k))|>0 \text {. }
$$

Лемма 11. Пусть $\left.n^{\prime}<n, n^{\prime} \geqslant\right] \log _{2} k[$. Тогдa

$$
L\left(n^{\prime}, k\right) \geqslant L(n, k)-3 k\left(\left(\frac{2}{3}\right)^{n^{\prime}}-\left(\frac{2}{3}\right)^{n}\right) .
$$

Доказательство. Обозначим через $\mathscr{D}_{\text {cut }}\left(n, n^{\prime}, k\right)$ множество деревьев высоты $n^{\prime}$ с $k$ концевыми вершинами над базисом $\left\{x_{1}^{s_{1}}, \ldots x_{n}^{s^{n}} \mid s_{i} \in\{0,1\}, i=1, \ldots, n\right\}$, через $\mathscr{D}\left(n, n^{\prime}, k\right)$ множество деревьев, корневые поддеревья высоты $n^{\prime}$ которых совпадают с деревьями из $\mathscr{D}_{c u t}\left(n, n^{\prime}, k\right)$, а из вершин яруса с номером $n^{\prime}$ выходят непересекающиеся цепочки длины $n-n^{\prime}$, при этом любая цепочка длины $n$, выходящая из корня дерева $D \in \mathscr{D}\left(n, n^{\prime}, k\right)$, содержит все переменные $x_{1}, \ldots, x_{n}$. Рассмотрим функщии $\varphi_{n, n^{\prime}}: \mathscr{D}\left(n, n^{\prime}, k\right) \rightarrow \mathscr{D}_{\text {cut }}\left(n, n^{\prime}, k\right)$, 
где функция $\varphi_{n, n^{\prime}}(D)$ выдает первые $n^{\prime}$ ярусов дерева $D$. Пусть $\mathscr{I}_{\max }(n, k) \subseteq \Phi(n, k)$ - такое множество, что если $I \in \Phi_{\max }(n, k), I^{\prime} \in \Phi(n, k)$, то $T(I) \geqslant T\left(I^{\prime}\right)$. Очевидно, что для $I_{1}, I_{2} \in \Phi_{\max }(n, k)$ справедливо равенство $T\left(I_{1}\right)=T\left(I_{2}\right)$. Также ясно, что для $I \in \Phi(n, k)$ справедливо равенство $L(n, k)=T(I)$.

Пусть $\oiint_{\max }(n, k)$ содержит такую задачу $I$, что ее оптимальное решающее ИД $D$ содержится в множестве $\mathscr{D}\left(n, n^{\prime}, k\right)$. Соответственно, любое другое дерево, находящееся в множестве $\mathscr{D}\left(n, n^{\prime}, k\right)$ и решающее ЗИП $I$, имеет большую сложность, чем дерево $D$. Каждое дерево $D^{\prime}$ из множества $\mathscr{D}\left(n, n^{\prime}, k\right)$ состоит из нижней части, равной $\varphi_{n, n^{\prime}}\left(D^{\prime}\right)$, и $k$ цепочек длины $n-n^{\prime}$. Сложность одной цепочки длины $n-n^{\prime}$, исходящей из вершины яруса с номером $n^{\prime}$ равна

$$
\left(\frac{2}{3}\right)^{n^{\prime}}\left(1+\frac{2}{3}+\ldots+\left(\frac{2}{3}\right)^{n-n^{\prime}-1}\right)=3\left(\left(\frac{2}{3}\right)^{n^{\prime}}-\left(\frac{2}{3}\right)^{n}\right)
$$

следовательно,

$$
T\left(D^{\prime}\right)=T\left(\varphi_{n^{\prime}, n}\left(D^{\prime}\right)\right)+3 k\left(\left(\frac{2}{3}\right)^{n^{\prime}}-\left(\frac{2}{3}\right)^{n}\right)
$$

Заметим, что все оптимальные решающие деревья для задач из множества $\mathscr{T}\left(n^{\prime}, k\right)$ находятся в множестве $\mathscr{D}_{c u t}\left(n, n^{\prime}, k\right)$, так как это множество содержит все деревья высоты $n^{\prime}$, решающие задачи из $\mathscr{I}\left(n^{\prime}, k\right)$. Кроме того,

$$
\max _{I \in \Phi\left(n^{\prime}, k\right)} T(I) \geqslant T\left(\varphi_{n, n^{\prime}}(D)\right)=T(D)-3 k\left(\left(\frac{2}{3}\right)^{n^{\prime}}-\left(\frac{2}{3}\right)^{n}\right) .
$$

Пусть не существует задачи из $\oiint_{\text {max }}(n, k)$, чье оптимальное решающее дерево принадлежит $\mathscr{D}\left(n, n^{\prime}, k\right)$. Это значит, что для любого дерева $D \in \mathscr{D}\left(n, n^{\prime}, k\right)$, решающего задачу из $\Im_{\text {max }}(n, k)$,

$$
T(D)>\max _{I \in \Phi_{\max }(n, k)} T(I) .
$$

Для каждой ЗИП из $\mathscr{I}_{\text {max }}(n, k)$ сушествует решаюшее дерево из $\mathscr{D}\left(n, n^{\prime}, k\right)$, например, сбалансированное дерево с характеристикой $] \log _{2} k[$, и его сложность больше, чем у оптимального дерева той же задачи. Следовательно,

$$
\max _{I \in \Phi\left(n^{\prime}, k\right)} T(I)>\max _{I \in \Phi(n, k)} T(I)-3 k\left(\left(\frac{2}{3}\right)^{n^{\prime}}-\left(\frac{2}{3}\right)^{n}\right) .
$$

Получаем, что при $\left.n^{\prime}<n, n^{\prime} \geqslant\right] \log _{2} k[$

$$
L\left(n^{\prime}, k\right) \geqslant L(n, k)-3 k\left(\left(\frac{2}{3}\right)^{n^{\prime}}-\left(\frac{2}{3}\right)^{n}\right) .
$$

\section{5. Поведение функции Шеннона}

Доказательство теоремы 1. Рассмотрим 5 случаев. 
1. Пусть $\log _{2} \log _{2} n=\bar{o}\left(\log _{2} k(n)\right)$ и $\log _{2} k(n) \lesssim c n, c<2 / 3$. Тогда из леммы 10 следует, что существует последовательность равномерно распределенных библиотек $V_{n}$ мощности $k(n)$ порядка

$$
s(n, k)=\log _{2} k(n)-\delta \log _{2}\left(16 \log _{2} k(n) \ln n\right)=\log _{2} k(n)+\bar{o}\left(\log _{2} k(n)\right), \quad \delta>1 .
$$

Из леммы 8 известно, что для ЗИП $I_{n}=\left\langle B_{3}^{n}, V_{n}, \rho\right\rangle$ с библиотекой из последовательности равномерно распределенных библиотек порядка $\log _{2} k(n)-\bar{o}\left(\log _{2} k(n)\right)$

$$
T\left(I_{n}\right) \gtrsim 6\left(\frac{4}{3}\right)^{\log _{2} k(n)+\bar{o}\left(\log _{2} k(n)\right)}=2^{\log _{2}(4 / 3)\left(\log _{2} k(n)+\bar{o}\left(\log _{2} k(n)\right)\right)} .
$$

Значит,

$$
\begin{aligned}
L(n, k) & \gtrsim 2^{\log _{2}(4 / 3)\left(\log _{2} k(n)+\bar{o}\left(\log _{2} k(n)\right)\right)} \\
\log _{2} L(n, k) & \gtrsim \log _{2} \frac{4}{3}\left(\log _{2} k(n)+\bar{o}\left(\log _{2} k(n)\right)\right) \sim \log _{2} \frac{4}{3} \log _{2} k(n) .
\end{aligned}
$$

2. Пусть $n \lesssim(3 / 2) \log _{2} k$ и $n \sim c \log _{2} k(n), c>1$. Возьмем $n_{1} \gtrsim c_{1} \log _{2} k, c_{1}>2 / 3$. Известно, что

$$
L\left(n_{1}, k\right) \gtrsim 2^{\log _{2}(4 / 3)\left(\log _{2} k(n)+\bar{o}\left(\log _{2} k\right)\right)},
$$

а из леммы 11 следует, что

$$
\begin{aligned}
L(n, k) & \geqslant L\left(n_{1}, k\right)-3 k\left(\left(\frac{2}{3}\right)^{n}-\left(\frac{2}{3}\right)^{n_{1}}\right) \\
& \gtrsim 2^{\log _{2}\left((4 / 3) \log _{2} k(n)(1+\bar{o}(1))\right)}-3 k\left(\left(\frac{2}{3}\right)^{c \log _{2} k(n)(1+\bar{o}(1))}-\left(\frac{2}{3}\right)^{n_{1}}\right) \\
& \geqslant 2^{\log _{2}\left((4 / 3) \log _{2} k(n)(1+\bar{o}(1))\right)}-3 k\left(\frac{2}{3}\right)^{c \log _{2} k(n)(1+\bar{o}(1))} \\
& =2^{\log _{2}\left((4 / 3) \log _{2} k(n)(1+\bar{o}(1))\right)}-2^{\left.\log _{2} k(n)+c \log _{2}(2 / 3) \log _{2} k(n)+\bar{o}\left(\log _{2} k(n)\right)\right)} \\
& =2^{\log _{2}(4 / 3)\left(\log _{2} k(n)+\bar{o}\left(\log _{2} k(n)\right)\right)}\left(1-2^{d(k, n)}\right),
\end{aligned}
$$

где

$$
\begin{aligned}
d(k, n) & =\log _{2} k(n)+c \log _{2} \frac{2}{3} \log _{2} k(n)-\log _{2} \frac{4}{3}\left(\log _{2} k(n)+\bar{o}\left(\log _{2} k\right)\right) \\
& =\log _{2} k(n)\left(1+c \log _{2} \frac{2}{3}-\log _{2} \frac{4}{3}+\bar{o}(1)\right) \\
& =\log _{2} k(n)\left(\log _{2} \frac{3}{2}-c \log _{2} \frac{3}{2}+\bar{o}(1)\right) \rightarrow-\infty
\end{aligned}
$$

при $n \rightarrow \infty, k(n) \rightarrow \infty$, так как $c>1$, и следовательно,

$$
L(n, k) \geqslant 2^{\log _{2}(4 / 3)\left(\log _{2} k(n)+\bar{o}\left(\log _{2} k(n)\right)\right)}(1+\bar{o}(1)) .
$$

Значит,

$$
\log _{2} L(n, k) \gtrsim \log _{2}(4 / 3)\left(\log _{2} k(n)+\bar{o}\left(\log _{2} k(n)\right)\right) \text {. }
$$


3. Пусть $\log _{2} k(n)=n-\bar{o}\left(\log _{2} k(n)\right)$. Тогда из леммы 6 следует, что

$$
L(n, k) \geqslant \frac{6 k}{2^{n}}\left(\left(\frac{4}{3}\right)^{n}-1\right)
$$

$\log _{2} L(n, k)=\log _{2} 6+\log _{2} k-n+\log _{2}\left(\left(\frac{4}{3}\right)^{n}-1\right)=\bar{o}\left(\log _{2} k\right)+\log _{2} \frac{4}{3} \log _{2} k$

Используя верхнюю оценку из следствия 1 , получаем, что если $n \rightarrow \infty, k(n) \rightarrow \infty$,

$$
\log _{2} \log _{2} n<\log _{2} k(n)-\log _{2} \log _{2} k(n), \quad \log _{2} k(n)=n-\bar{o}\left(\log _{2} k(n)\right),
$$

To

$$
\log _{2} L(n, k(n)) \sim \log _{2} \frac{4}{3} \log _{2} k(n)
$$

4. Пусть $k(n) \asymp 2^{n}$, то есть существует константа $c$ такая, что $k(n) \sim c 2^{n}$ при $n, k(n) \rightarrow \infty$. Из леммы 6 следует, что

$$
L(n, k) \geqslant \frac{6 k}{2^{n}}\left(\left(\frac{4}{3}\right)^{n}-1\right) \sim 6 c 2^{n \log _{2}(4 / 3)} \sim 6 c\left(\frac{k}{c}\right)^{\log _{2}(4 / 3)} \asymp k^{\log _{2}(4 / 3)} .
$$

Вместе с верхней оценкой (следствие 1) получаем, что при $k(n) \asymp 2^{n}$

$$
L(n, k(n)) \asymp(k(n))^{\log _{2}(4 / 3)} .
$$

5. Пусть $k \geqslant 2^{n-1}$, то есть $\left[\log _{2} k\right]=n-1$. Тогда из леммы 5 и следствия 1 следует, что нижняя оценка функции Шеннона совпадает с верхней и

$$
L(n, k)=6\left(\frac{4}{3}\right)^{n-1}+k\left(\frac{2}{3}\right)^{n-1}-6
$$

Теорема доказана.

В заключение автор выражает благодарность Э. Э. Гасанову за постановку задачи и научное руководство.

\section{Список литературы}

1. Блайвас Т. Д., Асимптотики задачи интервального поиска на булевом кубе в классе сбалансированных древовидных схем. Дискретная математика (2004) 16, №4, 65-78.

2. Гасанов Э. Э., Кудрявцев В. Б., Теория хранения и поиска информации. Физматлит, Москва, 2002.

3. Гасанов Э. Э., Оценки сложности одного метода решения задачи включающего поиска. Дискретная математика (2000) 12, № 2, 118-139. 